\title{
Selective One-Pot Synthesis of Trithiocarbonates, Xanthates and Dithiocarbamates for use in RAFT/MADIX Living Radical Polymerizations
}

\author{
Murray R. Wood ${ }^{\mathrm{a}}$, David J. Duncalf ${ }^{\mathrm{b}}$, Steven P. Rannard ${ }^{*, \mathrm{~b}}$, Sébastien Perrier*,a \\ ${ }^{a}$ Department of Colour and Polymer Chemistry, University of Leeds, Woodhouse Lane, Leeds, LS2 \\ 9JT, England \\ ${ }^{b}$ Unilever Research \& Development, Port Sunlight Laboratories, Quarry Road East, Bebington, \\ Wirral, L63 3JW, England
}

\section{Supporting information}

Characterisation data for 8 . Yield $=81 \% . \delta_{\mathrm{H}}\left(400 \mathrm{MHz}, \mathrm{CDCl}_{3}\right) \delta 4.62\left(\mathrm{~s}, 4 \mathrm{H}, \mathrm{PhCH}_{2}\right)$, $7.29\left(\mathrm{~m}, 10 \mathrm{H}, \mathrm{PhCH}_{2}\right),{ }^{13} \mathrm{C}-\mathrm{NMR}(100 \mathrm{MHz}) \delta 222.8,134.9,129.1,128.7,127.8$, 45.2, TOF-MS $\left(\mathrm{ES}^{+}\right) \mathrm{m} / z=291.034\left(\mathrm{MH}^{+}\right)$

Characterisation data for 9 . Yield $=64 \% . \delta_{\mathrm{H}}\left(400 \mathrm{MHz}, \mathrm{CDCl}_{3}\right) \delta 1.26-1.28(\mathrm{t}, J=7.2$ $\left.\mathrm{Hz}, 3 \mathrm{H}, \mathrm{OCH}_{3} \mathrm{CH}_{3}\right), 1.59-1.61$ (d, $\left.J=7.4 \mathrm{~Hz}, 3 \mathrm{H}, \mathrm{SCHCH}_{3}\right), 4.19-4.23$ (q, $J=3.7$ $\left.\mathrm{Hz}, 2 \mathrm{H}, \mathrm{OCH}_{2} \mathrm{CH}_{3}\right), 4.59-4.62\left(\mathrm{~s}, 2 \mathrm{H}, \mathrm{PhCH}_{2}\right), 4.78-4.83(\mathrm{q}, J=7.4 \mathrm{~Hz}, 1 \mathrm{H}$, $\left.\mathrm{SCHCH}_{3}\right), 7.25\left(\mathrm{~m}, 5 \mathrm{H}, \mathrm{PhCH}_{2}\right){ }^{13} \mathrm{C}-\mathrm{NMR}(100 \mathrm{MHz}) \delta 220.2,170.4,134.7,128.1$, $127.5,127.3,60.3,48.6,40.4,16.2,13.1$, TOF-MS $\left(\mathrm{ES}^{+}\right) \mathrm{m} / z=269.067\left(\mathrm{M}^{+}-\mathrm{S}\right)+\mathrm{H}^{+}{ }^{21}$

Characterisation data for 10. Yield $=68 \% . \delta_{\mathrm{H}}\left(400 \mathrm{MHz}, \mathrm{CDCl}_{3}\right) \delta 1.37(\mathrm{~s}, 6 \mathrm{H}$, $\mathrm{CH}_{3} \mathrm{CHCH}_{3}$ ), 4.32 (s, 2H, $\mathrm{PhCH}_{2} \mathrm{~S}$ ), 5.71-5.81 (m, $1 \mathrm{H}, \mathrm{CH}_{3} \mathrm{CHCH}_{3}$ ), 7.25-7.35 (m, $\left.5 \mathrm{H}, \mathrm{PhCH}_{2} \mathrm{~S}\right),{ }^{13} \mathrm{C}-\mathrm{NMR}(100 \mathrm{MHz}) \delta 213.15,135.8,129.4,128.7,127.5,43.2,40.3$, 21.5, TOF-MS $\left(\mathrm{ES}^{+}\right) \mathrm{m} / \mathrm{z}=227.056\left(\mathrm{MH}^{+}\right)$

Characterisation data for 11. Yield $=63 \% . \delta_{\mathrm{H}}\left(400 \mathrm{MHz}, \mathrm{CDCl}_{3}\right) \delta 1.67-1.69(\mathrm{~d}, J=$ $\left.7.3 \mathrm{~Hz}, 3 \mathrm{H}, \mathrm{SCHCH}_{3}\right), 4.17-4.23\left(\mathrm{q}, J=4.6 \mathrm{~Hz}, 1 \mathrm{H}, \mathrm{SCHCH}_{3}\right), 5.05-5.17$ (q, $J=14$ $\left.\mathrm{Hz}, 2 \mathrm{H}, \mathrm{PhCH}_{2}\right)$, 7.29-7.33 (m, 3H, $\mathrm{PhCH}_{2}$ ), 7.39-7.41 (d, J=6.1 Hz, 2H, $\left.\mathrm{CH}_{2} P h\right)$, ${ }^{13} \mathrm{C}-\mathrm{NMR}(100 \mathrm{MHz}) \delta 200.3,177.2,134.8,128.7,128.5,128.4,47.9,47.8,47.6$, 18.3, TOF-MS $\left(\mathrm{ES}^{+}\right) \mathrm{m} / \mathrm{z}=238.036\left(\mathrm{M}^{+}-\mathrm{O}\right)^{21}$

\footnotetext{
${ }^{21}$ As carbonyls and thiocarbonyls are particularly susceptible to fragmentation, the major peak in the MS analyses is assigned to the molecular ion, minus the carbonyl oxygen (sulfur). The nominal MS data show peaks with a lower intensity that do represent the expected molecular ion, but these peaks
} 
could not be analyzed with high resolution MS because of too much interferences from other fragments. The data is still correct and consistent with what could be expected from high resolution MS. 